\title{
The Impact of Cognitive Styles on Perceptual Distributed Multimedia Quality
}

\author{
Gheorghita Ghinea \\ Sherry Y. Chen \\ Department of Information Systems and Computing \\ Brunel University, Uxbridge, Middlesex \\ UB8 3 PH \\ \{George.Ghinea; Sherry.Chen\}@brunel.ac.uk
}

\begin{abstract}
Gheorghita Ghinea is Lecturer in the Department of Information Systems and Computing at Brunel University. He obtained the BSc (Honours) and MSc degrees in Computer Science from the University of the Witwatersrand, Johannesburg, South Africa, and the PhD degree, also in Computer Science, from the University of Reading in the United Kingdom. His research interests span multimedia (especially perpetual aspects), Quality of Service, as well as computer networking and security issues. He is a member of the IEEE. His home page is at http://www.brunel.ac.uk/ csstggg2.
\end{abstract}

Sherry Y. Chen is a Lecturer in the Department of Information Systems and Computing at Brunel University. Her major research interests focus on hypermedia-based learning environments and human-computer interaction. She has published widely in these areas. She obtained her $\mathrm{PhD}$ degree from University of Sheffield in the United Kingdom. She is the principle investigator of two research projects: "human factors in the design of adaptive hypermedia systems" and "cognitive approaches to develop search agents". Her home page is at http://www.brunel.ac.uk/ csstsyc.

\begin{abstract}
Multimedia technology has been widely used in the delivery of instructional material, but previous studies have indicated that individual differences, especially cognitive styles, have significant effects on users' preferences with respect to presentation of multimedia content. However, such research has thus far neglected to examine the effect of cognitive styles on users' subjective perceptions of multimedia quality. This study aims to examine the relationships between users' cognitive styles, the multimedia Quality of Service delivered by the underlying network, and users' Quality of Perception, which encompasses user levels of enjoyment and understanding of the informational content provided by multimedia material. Accordingly, 132 users took part in an experiment in which they were shown multimedia video clips presented with different values of two Quality of Service parameters (frame rate and colour depth). Results show that, whilst the two QoS parameters do not impact user QoP, multimedia content and dynamism levels significantly influence the user understanding and enjoyment component of QoP.
\end{abstract}




\section{Introduction}

In the past decade, the revolution in learning technology has transformed educational settings, placing prominence on the ability to deliver instructional material. New technologies in general, and multimedia in particular, play an increasingly important role in education (Schnotz and Lowe, 2003). Research in this area has mushroomed and shows several special concerns, one of which deals with the effectiveness of multimedia presentations (Garrett and Callear, 2001).

We believe that the effectiveness of a multimedia presentation would be hindered if it did not include the user experience in terms of enjoyment and information assimilation. Key to this is the issue of the quality of the multimedia presentation. Quality, in our perspective, has two main facets in a distributed multimedia environment: of service and of perception. The former, Quality of Service (QoS), illustrates the technical side of computer networking and represents the performance properties that the underlying network is able to provide. The latter, Quality of Perception (QoP), characterises the perceptual experience of the user when interacting with multimedia applications. Whilst the quality delivered by communication networks has traditionally been measured using QoS metrics, we believe that, as users are 'consumers' of multimedia applications, it is their opinions about the quality of multimedia material visualised which ultimately measures the success (or indeed, failure) of such applications to deliver desktop instruction material. When this delivery is done over Wide Area Networks such as the World Wide Web ('the Web'), transmission of multimedia data has to accommodate not only user subjective preferences, but also fluctuating networking environments.

The other concern is whether distributed multimedia presentations can accommodate individual differences. Previous studies indicate that users with different characteristics have different perceptions of multimedia presentation (Chen and Angelides, 2003). In particular, different cognitive style groups benefit from different types of multimedia presentation. Therefore, empirical evaluation that examines the impact of cognitive styles becomes paramount because such evaluations can provide concrete prescriptions for developing learner-centred systems that can match the particular needs of each cognitive style group. Whilst QoP has been investigated in 
the context of distributed multimedia quality (Ghinea and Thomas, 1998), the study did not take into account the possible effect of users' cognitive styles on their QoP. In this vein, the study presented in this paper aims to integrate QoP into the investigation of the perceptions of different cognitive style groups. Accordingly, the paper begins by building a theoretical background to present previous work done in the area of subjective distributed multimedia quality and to discuss the influence of cognitive styles on users' perception of multimedia presentations. It then progresses to describe and discuss the findings of an empirical study that investigated the relationships between cognitive styles and QoP. The paper ends with conclusions being drawn highlighting the value of integrating QoP when considering the impact of individual differences on users' perceptions of multimedia presentations.

\section{Theoretical Background}

\subsection{Quality of Service}

The networking foundation on which current distributed multimedia applications are built either do not specify Quality of Service (QoS) parameters (also known as best effort service) or specify them in terms of traffic engineering parameters such as delay, jitter, and loss or error rates. However, these parameters do not convey application-specific needs such as the influence of clip content and informational load on the user multimedia experience.

Furthermore, traditional approaches of providing Quality of Service (QoS) to multimedia applications have focused on ways and means of ensuring and managing different technical parameters such as delay, jitter and packet loss over unreliable networks. To a multimedia user, however, these parameters have little immediate meaning or impact. Although (s)he might be slightly annoyed at the lack of synchronisation between audio and video streams, it is highly unlikely that (s)he will notice, for instance, the loss of a video frame out of the 25 which could be transmitted during a second of footage, especially if the multimedia video in question is one in which the difference between successive frames is small. Moreover, in a distributed setting, the underlying communication system will not be able to provide an optimum QoS due to two competing factors - multimedia data sizes and network bandwidth. This results in phenomena such as congestion, packet loss, and errors. However, little 
work has been reported on the relationship between the network provided QoS and the satisfaction and perception of the user.

While the QoS impacts upon the perceived multimedia quality in distributed systems, previous work examining the influence of varying QoS on user perceptions of quality has almost totally neglected multimedia's edutainment quality (i.e., a mixture of both of educational as well as entertainment content), and has concentrated primarily on the perceived entertainment value of presentations displayed with varying QoS parameters.

Accordingly, previous work has studied the impact of varying clip frame rates on the user's enjoyment of multimedia applications (Apteker, 1995; Fukuda, 1997), and it has been shown that the dependency between human satisfaction and the required bandwidth of multimedia clips is non-linear. Consequently, a small change in human receptivity leads to a much larger relative variation of the required bandwidth. From a different perspective, Wijesekera and Srivastava (1996) and Wijesekera et al. (1999) have examined the effect that random media losses have on the user-perceived quality. Their work showed that missing a few media units will not be negatively perceived by a user, as long as too many such units are not missed consecutively and that this occurrence is infrequent. Moreover, because of the bursty nature of human speech (i.e. talk periods interspersed with intervals of silence), audio loss is tolerated quite well by humans as it results merely in silence elimination (21\% audio loss did not provoke user discontent, Wijesekera et al., 1999). However, viewer discontent for aggregate video losses increases gradually with the amount of losses, while for other types of losses and synchronisation defects there is an initial sharp rise in viewer annoyance that afterwards plateaus out.

Further work has been undertaken by Steinmetz (1996) who explored the bounds within which lip synchronisation can fluctuate without undue annoyance on the viewer's part, while the establishment of metrics for subjective assessment of teleconferencing applications was explored in Watson (1996, 1998). Indeed, the correlation between a user's subjective ratings of differing-quality multimedia presentation and physiological indicators has been studied by Wilson and Sasse 
(2000). However, research has largely ignored the influence that the user's psychological factors have on the perceived quality of distributed multimedia.

The focus of our research has been the enhancement of the traditional view of QoS with a user-level defined QoP. This is a measure that encompasses not only a user's satisfaction with multimedia clips, but also his/her ability to perceive, synthesise and analyse the informational content of such presentations. As such, we have investigated the interaction between QoP and QoS and its implications from both a user perspective as well as from a networking angle.

\subsection{Cognitive Styles}

Cognitive style is an individual's characteristic and consistent approach to organising and processing information. Riding and Rayner (1998) defined cognitive style as "an individual preferred and habitual approach to organising and representing information" (Riding and Rayner, 1998, p.25). Among a variety of cognitive styles, Field Dependence is related to the "degree to which a learner's perception or comprehension of information is affected by the surrounding perceptual or contextual field" (Jonassen and Grabowski, 1993, p. 87). Field Dependent people tend to perceive objects as a whole, whereas Field Independent people focus more on individual parts of the object. Field Dependent individuals rely more on external references; by contrast, Field Independent individuals rely more on internal references (Witkin et al., 1977).

Research has indicated that the dimensions of Field Dependence/-Independence have significant effects on individuals' preferences to multimedia presentations. Several studies suggested that Field Independent individuals could particularly get benefit from the control of media choice. A study by Chuang (1999) produced fourcourseware versions: animation+text, animation+voice, animation+text+voice, and free choice. The result showed that Field Independent subjects in the animation+text+voice group or in the free choice group scored significantly higher than those did in the animation+text group or in the animation+voice group. No significant presentation effect was found for the Field Dependent subjects. Similar results were obtained by Chanlin's (1998) study, which found Field Independent 
students did significantly better in visual control treatment, but there was no difference for Field Dependent students. Conversely, a number of studies have indicated that auditory cues are important to Field Dependent learners. Lee (1994) investigated the effectiveness of auditory cueing of interactive multimedia material. 87 students were randomly assigned to the auditory cueing group and non-auditory cueing group. The results showed that Field Dependent students would learn more effectively if the auditory cues were provided. Marrison and Frick (1994) also found similar results in a study conducted with 75 undergraduate students. The students were divided into three groups: multimedia instruction (MI), lecture with MI, and lecture only. Field Dependent students indicated that sound would enhance MI.

Results from the aforementioned studies suggest that different cognitive style groups have different preferences to multimedia presentation. These studies also indicate that further empirical work is needed to identify the preferences of different cognitive style groups, the results of which might help to guide the development and evaluation of multimedia information systems. This paper presents such a study, which aims to examine how cognitive styles influence users' responses to multimedia presentation when integrating the QoP metric.

\section{Methodology Design}

\subsection{Overview}

This study investigates the impact of cognitive styles on perceived multimedia quality. Among a variety of cognitive style characteristics, Witkin's Field Dependence was selected because it reflects how users organise information (Weller et al., 1994). Perceived multimedia quality was examined using the QoP measure, the only such metric that takes into account multimedia's edutainment duality. Accordingly, Figure 1 presents an overview of the experimental design of the study in a distributed multimedia context, applications are sent with various QoS parameters across networks such as the Web which, depending upon the bandwidth available to such applications, impact upon their presentation quality. This in turn is potentially perceived differently (from an informational as well as enjoyment perspective) by users, depending on their particular cognitive style. 


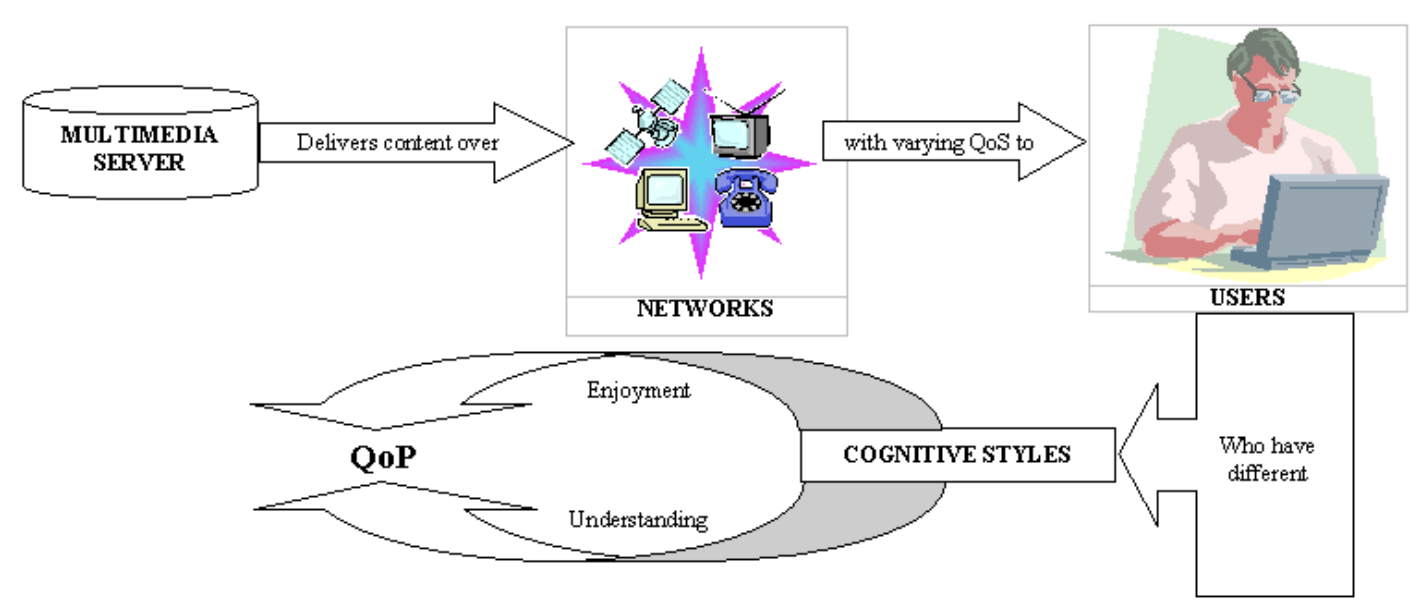

Figure 1: Experimental design of this study

\subsection{Participants}

This study was conducted at Brunel University's Department of Information Systems and Computing. 132 subjects participated in this study. Despite the fact that the participants volunteered to take part in the experiment, they were extremely evenly distributed in terms of cognitive styles, including 44 Field Independent users, 44 Intermediate users, and 44 Field Dependent users. In terms of gender, there were 70 male users and 62 female users. All of them were inexperienced in the content domain of the multimedia video clips visualized as part of our experiments, which will next be described next.

\subsection{Research Instruments}

\subsubsection{Video Clips}

A total of 12 video clips were used in our study. The multimedia clips were visualized under a Microsoft Internet Explorer browser with a Microsoft Media player plug-in, with users subsequently filling in a Web-based questionnaire to evaluate QoP for each clip.

These 12 clips had been used in previous QoP experiments (Ghinea and Thomas, 1998), and were between 30-44 seconds long and digitised in MPEG-1 format. The subject matter they portrayed was varied (as detailed in Table 1) and taken from 
selected television programmes, thereby reflecting educational and entertainment sources that average users might encounter in their everyday lives. Thus, six of the clips $(2,5,6,7,8$ and 12 in Table 1$)$ comprised predominantly educational content, with the remainder of the clips being viewed mainly for entertainment purposes. Also varied was the dynamism of the clips (i.e., the rate of change between the frames of the clip), which ranged from a relatively static news clip to a highly dynamic space action movie. Table 1 also describes the importance, within the context of each clip, of the audio, video and textual components as purveyors of information, as previously established through user tests (Ghinea and Thomas, 1998).

\begin{tabular}{|l|c|c|c|c|}
\hline VIDEO CATEGORY & Dynamic & Audio & Video & Text \\
\hline $\mathbf{1}$ - Action Movie & Strong & Medium & Strong & Weak/None \\
\hline 2 - Animated Educational Clip & Medium & Medium & Strong & Weak/None \\
\hline $\mathbf{3}$ - Band Clip & Medium & Strong & Medium & Weak/None \\
\hline $\mathbf{4}$ - Chorus Clip & Weak & Strong & Medium & Weak/None \\
\hline $\mathbf{5}$ - Computer Technology Clip & Medium & Strong & Strong & Medium \\
\hline $\mathbf{6}$ - Cooking Clip & Weak & Strong & Strong & Weak/None \\
\hline $\mathbf{7}$ - Documentary Clip & Medium & Strong & Strong & Weak/None \\
\hline $\mathbf{8}$ - News Clip & Weak & Strong & Strong & Medium \\
\hline $\mathbf{9}$ - Pop Music Clip & Medium & Strong & Strong & Strong \\
\hline $\mathbf{1 0}$ - Rugby Clip & Strong & Medium & Strong & Medium \\
\hline $\mathbf{1 1}$ - Snooker Clip & Weak & Medium & Medium & Strong \\
\hline $\mathbf{1 2}$ - Weather Forecast Clip & Weak & Strong & Strong & Strong \\
\hline
\end{tabular}

Table 1 Video Categories Used in Experiments

\subsubsection{Cognitive Style Analysis}

The cognitive style dimension investigated in this study was the level of Field Dependence. A number of instruments have been developed to measure Field Dependence, including the Group Embedded Figures Test (GEFT) by Witkin et al. (1971) and the Cognitive Styles Analysis (CSA) by Riding (1991). The GEFT derives scores for Field Independence by requiring subjects to locate simple shapes embedded in more complex geometrical patterns. However, a criticism of this approach is that levels of Field Dependence are inferred from poor Field Independence performance (Ford and Chen, 2000).

The CSA differs from the GEFT in that it includes two sub-tests. The first presents items containing pairs of complex geometrical figures that the individual is required to judge as either the same or different. The second presents items each comprising a 
simple geometrical shape, such as a square or a triangle, and a complex geometrical figure, as in the GEFT, and the individual is asked to indicate whether or not the simple shape is contained in a complex one by pressing one of two marked response keys (Riding and Grimley, 1999).

It seems that these two sub-tests have different purposes. The first sub-test is a task requiring Field Dependent capacity. Conversely, the second sub-test requires the disembedding capacity associated with Field Independence. In this way, the CSA overcomes the GEFT limitation that affects the measures of Field Dependence and Field Independence, because Field Dependent competence is positively measured rather than being inferred from poor Field Independent capability (Ford and Chen, 2000). In addition, the CSA offers computerized administration and scoring. Therefore, the CSA was selected as the measurement instrument for Field Dependence in this study.

The CSA measures what the authors refer to as a Wholist/Analytic (WA) dimension, noting that this is equivalent to Field Dependence/Independence (Riding and SadlerSmith, 1992). Riding's (1991) recommendations are that scores below 1.03 denote Field Dependent individuals; scores of 1.36 and above denote Field Independent individuals; students scoring between 1.03 and 1.35 are classed as Intermediate. In this study, categorisations were based on these recommendations.

\subsection{Procedure}

The experiment consisted of several steps. Initially, the CSA was used to classify users' cognitive styles as Field Independent, Intermediate, or Field Dependent. Subjects then viewed the 12 multimedia video clips. Each video clip was shown with a specific set of QoS parameters, unknown to the user. In our experiments, only the video stream QoS was targeted, since it is the video component which consumes most bandwidth in multimedia applications, and bandwidth is the most scarce networking resource in such environments. Accordingly, we varied the frame rate with which presentations were shown (video clips were displayed at 5, 15 or 25 frames per second -fps) and the colour depth (which could either be full 24-bit colour or a black and white presentation). A total of 22 users for each (frame rate, colour depth) combination were tested in the experiment. 
In order to counteract any order effects, the order in which clips were visualised was varied randomly for each participant. A between-subjects experimental design was chosen because this approach can easily identify how cognitive styles influence their perceptions of different frame rates and colour depths presented in multimedia video clips. After the users had seen each clip once, the window was closed, and they had to answer a number of questions about the video clip they had just seen. The actual number of such questions depended on the video clip, and varied between 10 and 12 . After the user had answered the set of questions pertaining to a particular video clip and the responses had been duly recorded, (s)he was asked to rate the enjoyment quality of the clip that had just been seen on a Likert scale of $1-6$ (with scores of 1 and 6 representing the worst and, respectively, best perceived qualities possible). The user then went on and watched the next clip.

Users were instructed not to let personal bias towards the subject matter in the clip or production-related preferences (for instance the way in which movie cuts had been made) influence their enjoyment quality rating of a clip. Instead, they were asked to judge a clip's enjoyment quality by the degree to which they, the users, felt that they would be satisfied with a general purpose multimedia service of such quality. Users were told that factors which should influence their quality rating of a clip included clarity and acceptability of audio signals, lip synchronisation during speech, and the general relationship between visual and auditory message components

\subsection{Data Analyses}

In this study, the independent variables include the participants' cognitive styles, as well as clip categories, degree of dynamism, frame rates, and colour depth. The dependent variables were the two components of Quality of Perception: the level of understanding (expressed as a percentage measure describing the proportion of questions that the user had correctly answered for each clip) as well as the level of enjoyment (expressed on a 6-point Likert scale). Data were analysed with the Statistical Package for the Social Sciences (SPSS) for Windows version (release 9.0). An ANalysis Of VAriance (ANOVA), suitable to test the significant differences of three or more categories, and t-test, suitable to identify the differences between two 
categories (Stephen and Hornby, 1997), were applied to analyse the participants' responses. A significance level of $p<0.05$ was adopted for the study.

\section{Discussion of Results}

\subsection{Subject Content}

Our analysis has highlighted that the subject content (i.e., particular clip category) has a statistically significant impact on the level of participants' understanding $(p=.0000)$. This confirms previous results (Ghinea and Thomas, 1998) and we extended our analysis to include the impact of users' cognitive styles. As depicted in Table 2, the clip on which participants performed best varied according to the users' cognitive style. Accordingly, Intermediate and Field Independent users had the highest level of understanding for the Snooker video clip. However, Field Dependent users perform better in the Documentary clip. As Table 1 shows, the most discriminating feature between the two clips is the fact that the latter clip does not contain any textual description. The difference in the understanding corresponding to the different cognitive styles is thus probably due to the fact that Field Dependent learners are “influenced by salient features" (Jonassen and Grabowski, 1993, p.88). Without the distraction of text description, Field Dependent users could concentrate their learning on video clips, so they could have better performance. On the other hand, irrespective of the type of cognitive style, all users performed worst in the highly dynamic Rugby sports action clip, in which, as Table 1 shows, all media components were MediumStrong purveyors of information. This finding seems to imply that users have difficulty concentrating on multiple, different sources of information, and that the dynamism of the clip is also a contributing factor to participants' level of understanding, as was confirmed by further analysis, presented in section 4.2.

\begin{tabular}{|l|c|c|c|}
\hline & Field Dependent & Intermediate & Field Independent \\
\hline Best Performance & Documentary & \multicolumn{2}{|c|}{ Snooker } \\
\hline & $63.84 \%$ & $62.93 \%$ & $63.46 \%$ \\
\hline Worst Performance & & Rugby \\
\hline & $33.24 \%$ & $37.5 \%$ & $34.82 \%$ \\
\hline
\end{tabular}

Table 2: Cognitive Styles and Level of Understanding in Clip Categories 
The specific multimedia clip type also influences the second component of QoP, namely the level of enjoyment experienced by users ( $\mathrm{p}=.0000)$. As Table 3 shows, although the Documentary and Rugby video clips predominate in the "Most Enjoyed" and "Least Enjoyed" categories, only for Field Dependent users does the choice of most/least enjoyed clip coincide with the clips on which the level of understanding is highest, respectively lowest (see Table 2, in this regard). These results are in line with those of Fullerton (2000) and Ford and Chen (2001), which showed that Field Dependent users performed better in a learning environment matching their preferences; conversely, their performance will be reduced in a mismatched condition. Moreover, our results also show that the Forecast video clip was the one which Field Independent users enjoyed most. It is probable that the wealth of detail present in this clip (information was conveyed through all three channels, video, audio and text) is what makes the clip appealing to this particular type of users, who concentrated primarily on procedural details when processing information in a learning context (Pask, 1976, 1979). This is in contrast to the "Most Enjoyed" clip (i.e. Documentary) for the other two categories of users, in which information was only conveyed through the video and audio streams.

\begin{tabular}{|l|c|c|c|}
\hline & Field Dependent & Intermediate & Field Independent \\
\hline Most Enjoyed & \multicolumn{2}{|c|}{ Documentary } & Forecast \\
\hline & 3.18 & 2.86 & 3.02 \\
\hline Least Enjoyed & Rugby & Band & Rugby \\
\hline & 1.39 & 1.93 & 1.61 \\
\hline
\end{tabular}

Table 3: Cognitive Styles and Enjoyment of Clip Categories

\subsection{Degree of Dynamism}

Multimedia clip dynamism was found to significantly impact upon participants' level of understanding in our study (Figure 2). The level of significance was found to be $\mathrm{p}=.000$ for Field Dependent users and $\mathrm{p}=.001$ for Intermediate and Field Dependent users. Clip dynamism is given by Table 1, where the terms strong, medium and weak were coded with the values of 3,2, and 1, respectively. All users performed worst in the clips with strong dynamism. In particular, Field Dependent users do not perform as well as Field Independent and Intermediate users. As suggested by previous works (Chen and Macredie, 2002; Chen, 2002), Field Dependent users' performance was hindered in situations where they need to extract cues by themselves. Thus, in 
multimedia clips with strong dynamism that provided too many cues, Field Dependent users might find it difficult to select relevant cues.

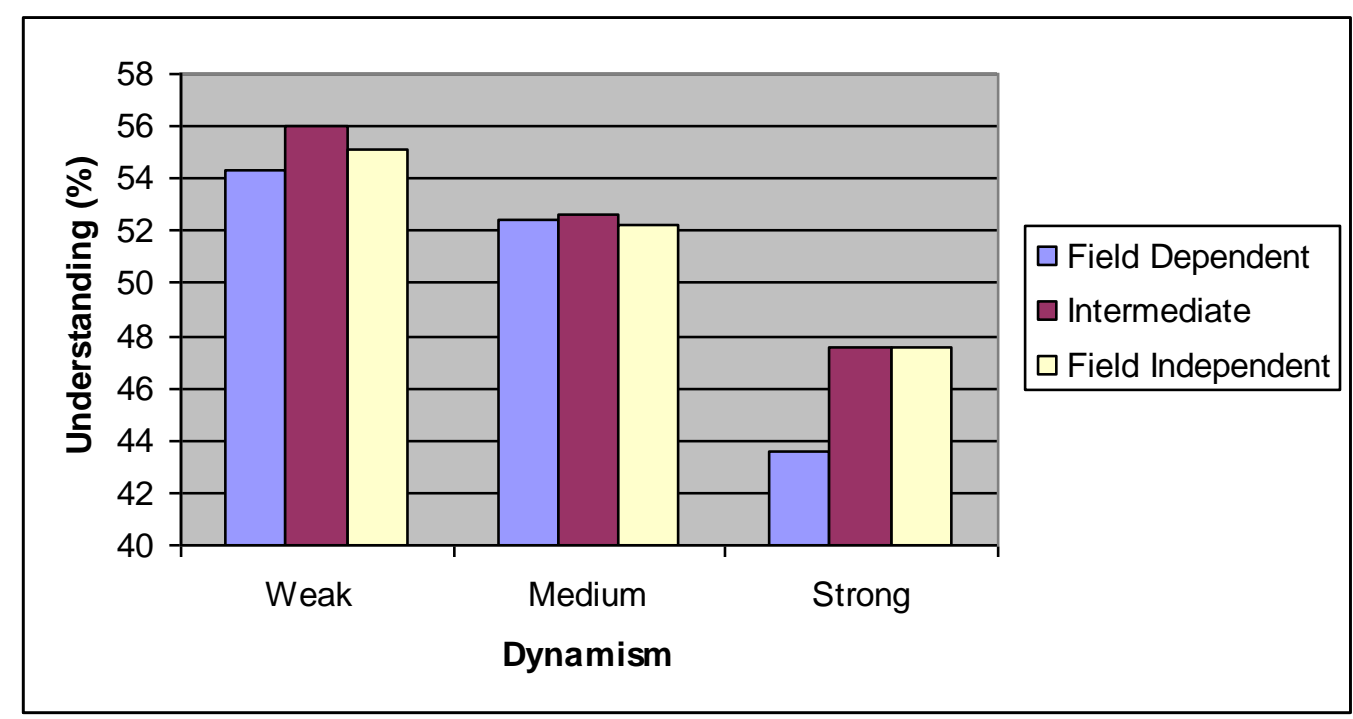

Figure 2 Impact of Dynamism and Cognitive Styles on Participants' Level of Understanding

The dynamism of the visualized clips also influenced the level of enjoyment experienced by participants $(\mathrm{p}=.000)$. If a per-cognitive style analysis is pursued, we find that the level of enjoyment is influenced by the dynamism of the multimedia clip for both Field Independent ( $\mathrm{p}=.004)$ and Field Dependent $(\mathrm{p}=.000)$ users. As shown in Figure 3, both Field Independent and Field Dependent users experienced higher levels of enjoyment from the clips with medium dynamism, while strongly dynamic clips were liked least of all. However, dynamism does not seem to be a factor influencing multimedia clip enjoyment of Intermediate users. One possible interpretation is that individuals possessing an Intermediate cognitive style employ a more versatile repertoire of information seeking strategies. Versatile users, who have acquired the skill to move back and forth between different information seeking strategies, are more capable of adapting themselves to suit the subject content presented by the multimedia video clips. This finding is consistent with the views of previous work, namely that a versatile strategy can be better equipped for multimedia learning technology (Chen and Ford, 1998; Paterson, 1996). 


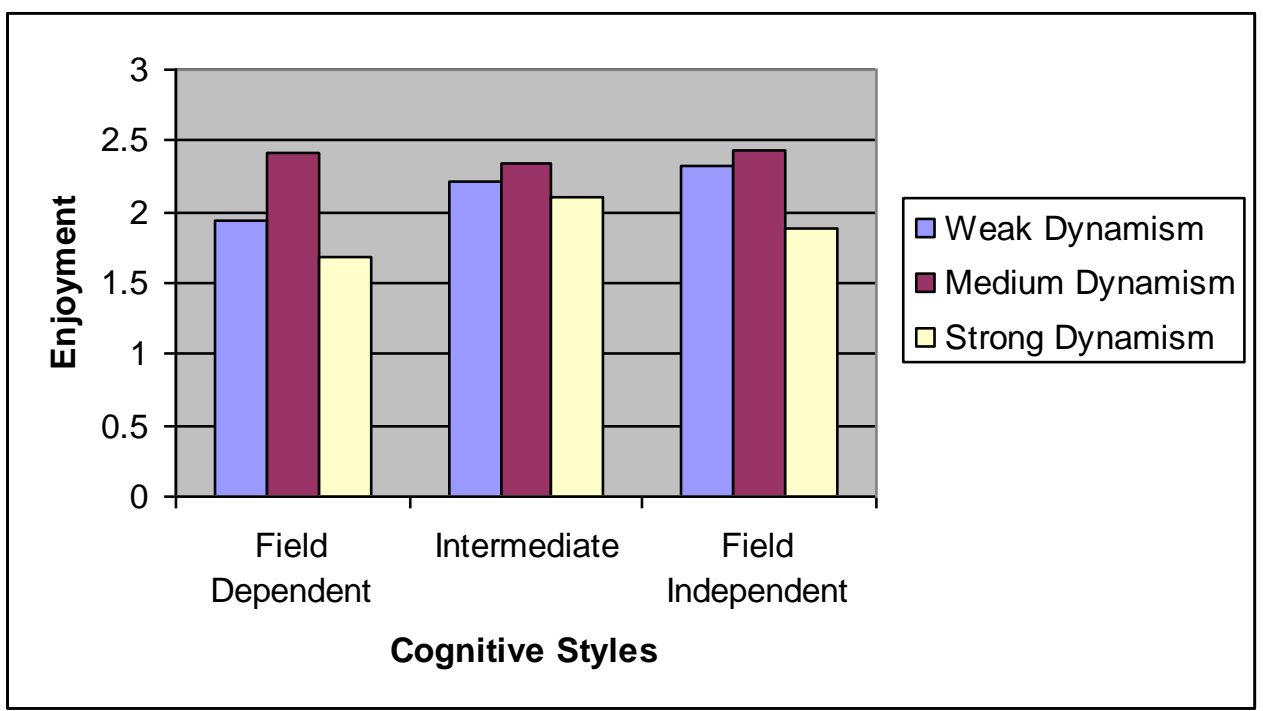

Figure 3 Impact of Dynamism and Cognitive Styles on Participants' Enjoyment

\subsection{Frame Rate and Colour Depth}

The results indicated that frame rate and colour depth did not influence users' levels of understanding and levels of enjoyment for the whole sample. Likewise, there was no significant effect of these factors for each cognitive style group. This finding has considerable implications in environments such as the Web, where numerous multimedia applications compete for (scare) bandwidth, for it shows that there is no significant difference in participants' QoP when viewing a presentation at full quality (25fps, 24-bit colour) or with seriously degraded parameters (5fps, black and white). Moreover, this finding occurs irrespective of cognitive style. Thus, substantial bandwidth savings can be obtained without a significant effect on participants' level of understanding and enjoyment of multimedia applications.

Moreover, in contrast to previous findings (Apteker et al., 1995), which showed that there was a dependency between users' enjoyment of multimedia applications and the frame rate with which they were viewed, our results would seem to indicate that when viewing a multimedia clip for educational/informational purposes as well, any degradations in quality are not noticed or are ignored by users.

\section{Conclusion}

This paper has presented the results of a study that looked at the impact of cognitive styles and Quality of Service parameters on users' subjective Quality of Perception. 
The latter is a term which encompasses not only a user's enjoyment, but also his/her level of understanding of visualized multimedia content. The results of this study indicated that multimedia content and dynamism level significantly impacted QoP. This would imply that in order to deliver an enhanced multimedia edutainment experience, educators should focus on relatively static multimedia video and take into consideration the appropriateness of the subject matter for the purposes of the specific educational objectives being sought.

One of our interesting findings also highlights that bandwidth-constrained environments, such as the Web, do not necessarily seem to have a negative impact on the user's educational experience, since, in our experiments, this does not suffer any significant change, irrespective of the technical quality with which a multimedia application is shown in such settings. Moreover, this finding occurs independently of participants' cognitive style. Whilst we agree that there are a host of other factors that come into the educational experience - delivered technical quality interplay, our findings nevertheless do open up new avenues for future research in this field.

This study has provided evidence of the benefits of integrating, in a multimedia edutainment setting, subjective QoP, the QoS with which multimedia presentations could be shown, and users' cognitive styles. However, our work is a small step. There is a need to conduct future research that would examine the impact of other individual differences such as. gender difference, prior knowledge, or culture background. Such research could also be conducted within a more sophisticated 3D multimedia environment, as it would be interesting to see how different cognitive style groups perceive multimedia features in such a setting. The ultimate goal of such studies would be to integrate their findings to build robust user models for the development of personalised multimedia environments that can accommodate users' individual differences into truly end-to-end communication architectures.

\section{Acknowledgements}

The authors wish to acknowledge the help provided by Sundeep Gupta, who performed the empirical study described in this paper. 


\section{References}

Apteker, R.T., Fisher, J.A., Kisimov, V.S. and Neishlos, H. (1995) Video Acceptability and Frame Rate. IEEE Multimedia 2 (3) 32-40.

ChanLin, L. (1998) Students' cognitive styles and the need for visual control in animation. Journal of Educational Computing Research 19 (4) 351-363.

Chen, S. Y. and Angelides, M. C. (2003) Customisation of Internet multimedia information systems design through user modelling, in: Architectural Issues of Web-Enabled Electronic Business, Shi Nansi Ed., Idea Group Publishing, 241-255.

Chen, S. Y. (2002) A Cognitive Model for Non-linear Learning in Hypermedia Programmes . British Journal of Educational Technology 33 (4) 453-464.

Chen, S. Y. and Ford, N. J. (1998) Modelling User Navigation Behaviours in a Hypermedia-Based Learning System: An Individual Differences Approach. International Journal of Knowledge Organization 25 (3) 67-78.

Chen, S. Y. and Macredie, R. D. (2002) Cognitive Styles and Hypermedia Navigation: Development of A Learning Model. Journal of the American Society for Information Science and Technology 53 (1) 3-15.

Chuang, Y-R. (1999) Teaching in a Multimedia Computer Environment: A study of effects of learning style, gender, and math achievement. Available: http://imej.wfu.edu./articles/1999/1/10/index/asp [21/12/2001]

Ford, N. and Chen, S. Y. (2001) Matching/Mismatching Revisited: An Empirical Study of Learning and Teaching Styles. British Journal of Educational Technology 32 (1) 5-22.

Ford, N. and Chen, S. Y. (2000) Individual differences, hypermedia navigation and learning: an empirical study. Journal of Educational Multimedia and Hypermedia 9 (4) 281-312.

Fukuda, K., Wakamiya, N., Murata, M., and Miyahara, H. (1997) QoS Mapping between User's Preference and Bandwidth Control for Video Transport, Proceedings of the 5th International Workshop on QoS (IWQoS) 291 - 301

Fullerton, K. (2000) The interactive effects of field dependence-independence and Internet document manipulation style on student achievement from computerbased Instruction. Ed.D Dissertation. University of Pittsburgh.

Garrett , B. M. and Callear, D. (2001) The value of intelligent multimedia simulation for teaching clinical decision-making skills. Nurse Education Today 21(5) $382-390$

Ghinea, G. and Thomas, J.P. (1998) QoS Impact on User Perception and Understanding of Multimedia Video Clips, Proceedings of ACM Multimedia '98 49 - 54.

Jonassen, D. H. and Grabowski, B. (1993) Individual Differences and Instruction. New York: Allen \& Bacon. 
Lee, C. H. (1994) The Effects of Auditory Cues in Interactive Multimedia and Cognitive Style on Reading Skills of Third Graders, Unpublished Ed.D. Dissertation, University of Pittsburgh, USA.

Marrison, D. L. and Frick, M. J. (1994) The Effect of Agricultural Students' Learning Styles on Academic Achievement and Their Perceptions of Two Methods of Instruction. Journal of Agricultural Education, 35 (1) 26-30

Pask, G. (1976). Styles and strategies of learning, British Journal of Educational Psycholog, 46, 128-48.

Pask, G. (1979). Final report of S.S.R.C. Research Programme HR 2708. Richmond (Surrey): System Research Ltd.

Paterson P. (1996). The influence of learning strategy in a computer mediated learning environment Paper presented at ALT-Conference '96. Available: http://www.warwick.ac.uk/alt-/alt-96/papers.html [19/11/1997]

Riding, R. J. (1991) Cognitive Styles Analysis, Birmingham: Learning and Training Technology.

Riding, R.J. and Grimley, M. (1999) Cognitive style and learning from multimedia materials in 11 year old children. British Journal of Educational Technology 30 (2) $43-56$.

Riding, R.J. and Rayner, S.G. (1998) Cognitive Styles and Learning Strategies, London: David Fulton Publisher.

Schnotz , W. and Lowe, R. (2003) External and internal representations in multimedia learning, Learning and Instruction 13 (2) 117-123

Steinmetz, R. (1996) Human Perception of Jitter and Media Synchronisation. IEEE Journal on Selected Areas in Communications 14 (1) 61 -72.

Stephen, P. and Hornby, S. (1997) Simple Statistics for Library and Information Professionals. London: Library Association.

Weller, H. G., Repman, J., and Rooze, G. E. (1994) The Relationship of learning, behavior, and cognitive styles in hypermedia-based instruction: Implications for design of HBI. Computers in the Schools 10, 401-420.

Wijesekera, D. and Srivastava, J. (1996) Quality of Service (QoS) Metrics for Continuous Media, Multimedia Tools and Applications 3 (1) 127-136.

Wijesekera, D., Srivastava, J., Nerode, A., and Foresti, M. (1999) Experimental Evaluation of Loss Perception in Continuous Media, Multimedia Systems 7 (6) 486-499.

Wilson, G. M. and Sasse, M. A. (2000) Investigating the Impact of Audio Degradations on Users: Subjective vs. Objective Assessment Methods. Proceedings of OZCHI'2000, Sydney, Dec. 2000. pp. 135-142

Witkin, H. A., Moore, C. A., Goodenough, D. R., and Cox., P. W. (1977) Fielddependent and field independent cognitive styles and their educational implications. Review of Educational Research, 47, 1-64.

Witkin, H. A., Oltman, P. K., Raskin, E., and Karp, S. A. (1971) A Manual For The Group Embedded Figures Test. Palo Alto, CA: Consulting Psychologists Press. 